\title{
A Lack of Correlation between the Incidence of Lyme Disease and Deaths due to Alzheimer's Disease
}

\author{
Danton H. O'Day ${ }^{\mathrm{a}, \mathrm{b}, *}$ and Andrew Catalano ${ }^{\mathrm{c}}$ \\ ${ }^{a}$ Department of Biology, University of Toronto at Mississauga, Mississauga, ON, Canada \\ ${ }^{\mathrm{b}}$ Department of Cell and Systems Biology, University of Toronto, Toronto, ON, Canada \\ ${ }^{\mathrm{c}}$ Department of Chemistry, City College of New York, New York, NY, USA
}

Accepted 25 March 2014

\begin{abstract}
Reports that Lyme disease (LD) causes Alzheimer's disease (AD) have appeared in academic journals and online. If the biological agent Borrelia burgdorferi that causes LD also causes AD, then areas with the highest levels of LD should have significantly higher numbers of deaths due to AD compared to low LD areas. Here we show there is no statistically significant correlation between the incidence of LD and deaths due to AD in the US. Furthermore, the 13 states with the highest deaths due to $\mathrm{AD}$ were statistically different $(p<0.0001)$ from those with high $\mathrm{LD}$ incidence.
\end{abstract}

Keywords: Alzheimer's disease, Borrelia burgdorferi, disease incidence, lyme disease

\section{INTRODUCTION}

Alzheimer's disease (AD) is a neurodegenerative disease with no proven initiating cause. Currently over $5,000,000$ individuals in the US have the disease, which is the sixth major cause of death [1]. Understanding the causes of $\mathrm{AD}$ is a medical priority. Lyme disease (LD), which leads to the neurodegenerative disease neuroborreliosis, is caused by the bacterium Borrelia burgdorferi [2]. Independent research groups have presented different lines of evidence arguing that LD can cause AD [3-6]. Thus there are valid reasons to believe that $\mathrm{LD}$ could cause AD. B. burgdorferi is a member of the spirochete family of bacteria, and spirochetes such as Treponema pallidum can cause dementia [7]. A diversity of postmortem techniques (e.g., ultrastructural visualization, immunolocalization, western blot,

\footnotetext{
*Correspondence to: Danton H. O’Day, Department of Biology, University of Toronto at Mississauga, 3359 Mississauga rd. N., Mississauga, ON, L5L 1C6, Canada. E-mail: danton.oday @utoronto.ca.
}

RNA sequence analysis, ELISA, PCR, etc.) have suggested the presence of various spirochetes, including B. burgdorferi, in the brains of AD individuals [3-5, 8]. The relationship between $\mathrm{LD}$ and $\mathrm{AD}$ has also been supported by analysis of existing data using Koch's and Hill's Postulates [8]. What is lacking to fulfill these postulates is the isolation of functional entities (e.g., B. burgdorferi) followed by proof of their ability to induce AD-related events in tissue culture cells.

Two hallmarks of AD are the build-up of plaques and tangles in the brain which are linked to neurodegenerative events $[9,10]$. Borrelia burgdorferi can induce both amyloid- $\beta$ and phosphorylated tau formation in tissue culture cells [5]. Thus accumulating circumstantial evidence does suggest a link between $\mathrm{LD}$ and AD. A quick Internet search reveals that this link has spawned widespread concern (e.g., [11, 12]). The ongoing interest and concern makes it critical to validate or disprove any relationship between the two diseases. Here we test a simple hypothesis: If LD does cause $\mathrm{AD}$, then the incidence of deaths due to $\mathrm{AD}$ 
Table 1

Two-by-two contingency table of the states with high and low Alzheimer's disease (AD) levels that also exhibit either high or low Lyme disease (LD) levels.

\begin{tabular}{lcc}
\hline & High LD & Low LD \\
\hline High AD & 1 & 7 \\
Low AD & 7 & 2 \\
\hline
\end{tabular}

Fisher's exact test two-tailed $p$-value $=0.015$. This statistically significant difference indicates a correlation between high $\mathrm{AD}$ and low $\mathrm{LD}$ and/or low $\mathrm{AD}$ and high $\mathrm{LD}$, demonstrating that high $\mathrm{AD}$ is not correlated with high LD.

should higher where LD is most prevalent compared to low $\mathrm{LD}$ areas.

\section{MATERIALS AND METHODS}

To our knowledge, location-specific data for the reporting of cases of both $\mathrm{LD}$ and deaths by $\mathrm{AD}$ is only available for the US. The age-adjusted death rates for $\mathrm{AD}$ were taken from Fig. 4 in Tejada [13]. The data for the reported cases of LD by state (2002-2011) were from the Centers of Disease Control and Prevention website (June 25, 2013 [14]). Although many factors affect the accuracy of the disease including the consistency of reporting of $\mathrm{LD}$ and deaths $\mathrm{AD}$, which will varies between states, it is assumed that this variability is negated by the comparison of 13 different states for each disease. The data sets used were the best and most current available at the time of writing. Means and standard deviations were calculated for sets of 13 states: those with the highest incidence of LD (18.978-68.222 cases/1000) and those with low to non-existent (0.0-0.189) LD. The Mann-Whitney U test was used to determine if $\mathrm{AD}$ is more prevalent in states with high LD compared to those with low LD. $\mathrm{AD}$ incidence in the 13 states with the highest LD incidence (MN, MD, VT, NY, WI, RI, ME, PA, NJ, MA, $\mathrm{NH}, \mathrm{DE}$, and CT) was compared with AD incidence in the 13 states with the lowest LD incidence (CO, AR, OK, LA, AZ, MS, NM, UT, GA, AL, SD, MT, and WA). Hawaii, which has a zero incidence of LD, was excluded because it is a distinct entity outside of the contiguous US states. Fisher's exact test was used to determine if the 13 states with the highest AD incidence (LA, VT, CO, AL, MS, IA, KY, SC, AZ, SD, $\mathrm{ND}, \mathrm{TN}$, and WA) were also states with high LD incidence. Fisher's exact test was also used to determine if high $\mathrm{AD}$ was correlated with high LD in a two-by-two contingency table (Table 1).

\section{RESULTS}

The mean value for the incidence of LD was calculated for each state and plotted beside AD rates (Fig. 1). With the exception of Vermont, the 13 highest LD

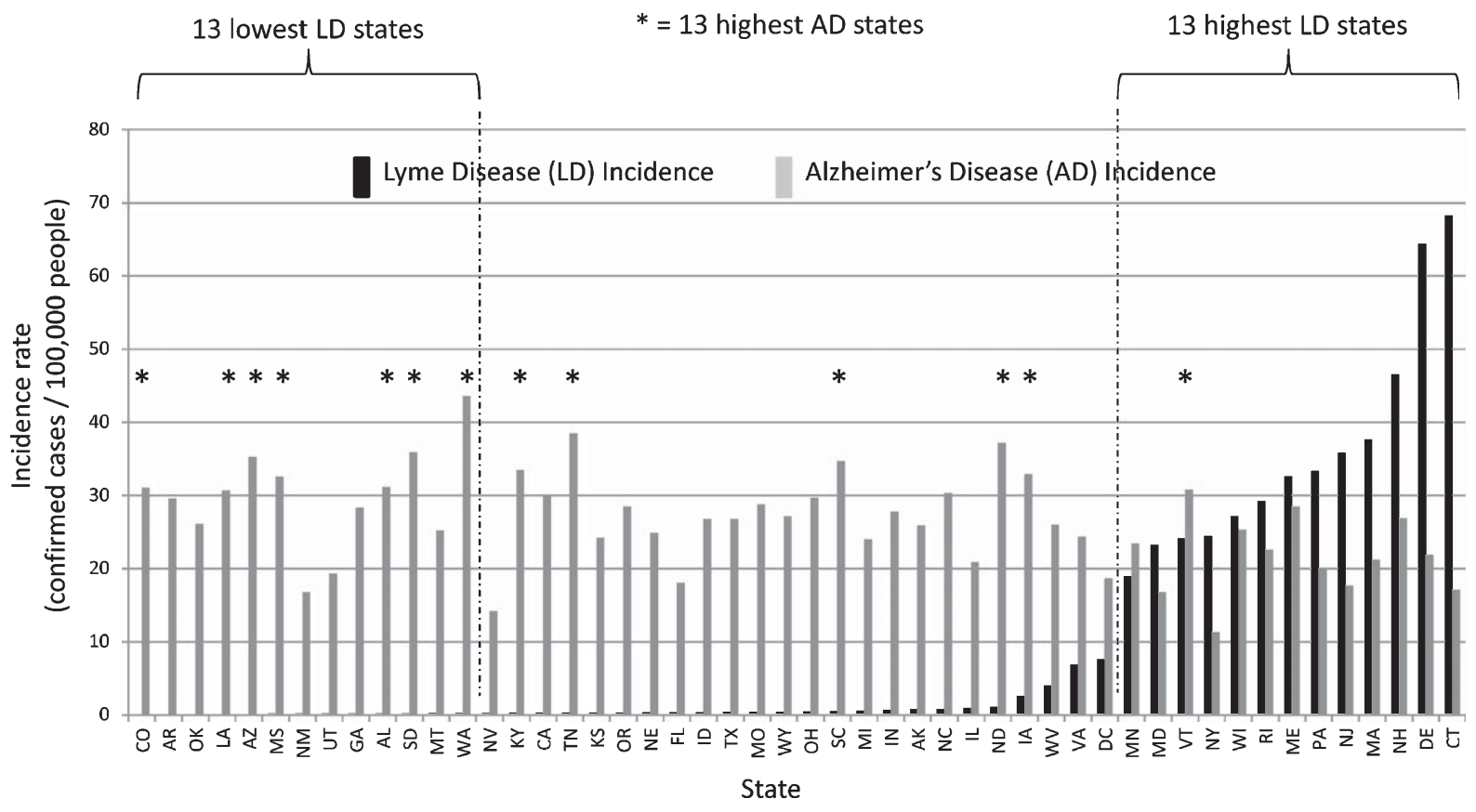

Fig. 1. Incidence of Lyme disease (LD, black) and of deaths caused by Alzheimer's disease (AD, grey) by U.S. state. States are ordered by increasing LD rate. The 13 highest and lowest LD rates (brackets) and 13 highest AD rates (*) are indicated. 


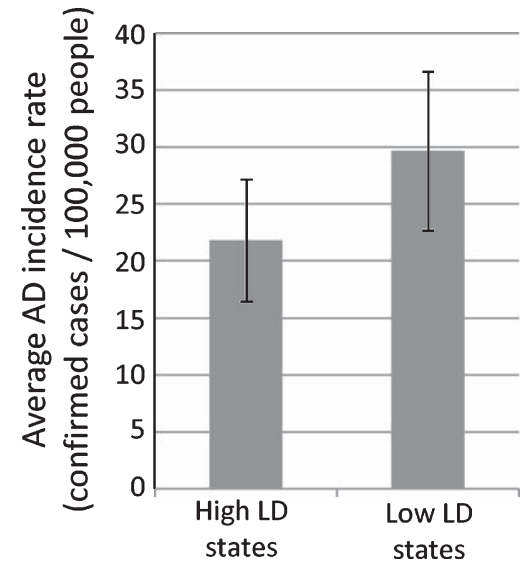

Fig. 2. Deaths by Alzheimer's disease (AD) rate in high versus low Lyme disease (LD) states.

states are different from the 13 highest AD states. In fact, 7 of the 13 highest LD states fall within the 13 states with the lowest incidence of deaths due to AD. We tested these observations against the assumption that if $\mathrm{LD}$ and $\mathrm{AD}$ were correlated, they should share most if not all of the high incidence states in common. The results of the statistical analysis reveal the 13 highest AD states are different from the 13 highest LD states $(p<0.0001)$. To determine if there is any correlation between AD and LD, the annual incidence of deaths due to AD were compared between U.S. states which had the highest and lowest incidences of LD. Although AD incidence was slightly greater in states with low $\mathrm{LD}$, this difference was not significant. Therefore, AD is not more prevalent in high LD states (Fig. 2). Taken one step further, Table 1 presents a two-by-two contingency table of high and low AD states versus high and low LD states. Fisher's exact test resulted in a twotailed $p$-value of 0.015 indicating a correlation between either low $\mathrm{AD}$ and high $\mathrm{LD}$ and/or high $\mathrm{AD}$ and low LD. This means high $\mathrm{AD}$ is not correlated with high LD.

\section{DISCUSSION}

If $B$. burgdorferi causes $\mathrm{AD}$, then areas with the highest incidence of LD should also have significantly higher numbers of deaths due to AD. A simple graphic presentation of the incidence of LD state-by-state in the US versus the incidence of deaths due to AD clearly reveals a lack of co-incidence between these two variables. When deaths due to AD in 13 of the states with the highest incidence of LD were compared with those with the lowest, there is no statistically significant dif- ference between them. Furthermore, if $\mathrm{LD}$ and $\mathrm{AD}$ are correlated, they would have most of the high incidence states in common. However, the 13 highest AD states are significantly different $(p<0.0001)$ from the 13 highest LD states. Moreover, Table 1 demonstrates not only a lack of correlation but rather an association between high AD and low LD suggesting one disease excludes the other. However due to the small numbers, we interpret the data as simply showing no relationship between the two diseases. The lack of correlation between the incidence of LD and AD on a state-by-state basis and the lack of any demonstrated relationship between the two diseases argue there is no link between them. In support of this, Krut et al. [15] examined the levels of AD-specific biomarkers in cerebrospinal fluid revealing that the neuroborreliosis profiles were different from the AD profiles, suggesting some of the early correlative studies between the two diseases may not be accurate.

It is important to recognize that $\mathrm{LD}$ and $\mathrm{AD}$ are two distinct diseases with different latency periods. Although LD has a predominant effect on young males aged 5-9, the disease significantly affects individuals of all ages [16]. While it might seem that a disease affecting young people might not translate to an effect in aging individuals, recent research has revealed that the cause(s) of AD occur decades before any symptoms of the disease manifest themselves [10].

The authors have used the best data available to evaluate the proposed link between LD and AD. This does not mean that these data are without issues including consistency of data collection between states and the two diseases, among other variables. What the data do reveal is that there is no evidence to argue that these two diseases are in any way related which hopefully will be communicated to those who have concerns about the relationship between $\mathrm{LD}$ and AD.

\section{ACKNOWLEDGMENTS}

Robert Huber is thanked for critical comments. Natural Sciences and Engineering Research Council research grant (A6807; DO'D).

Authors' disclosures available online (http://www.jalz.com/disclosures/view.php?id=2233).

\section{REFERENCES}

[1] Alzheimer's Association (2013) Alzheimer's Disease Facts and Figures, http://www.alz.org/alzheimers_disease_facts_and figures.asp, Last updated 2014, Accessed on February 26, 2014. 
[2] van Dam AP, Kuiper H, Vos K, Widjojokusumo A, de Jongh BM, Spanjaard L, Ramselaar AC, Kramer MD, Dankert J (1993) Different genospecies of Borrelia burgdorferi are associated with distinct clinical manifestations of Lyme borreliosis. Clin Infect Dis 17, 708-717.

[3] MacDonald AB (1988) Concurrent neocortical Borreliosis and Alzheimer's-demonstration of a spirochetal cyst form. Ann N Y Acad Sci 539, 468-470.

[4] Meer-Scherrer L, Chang Loa C, Adelson ME, Mordechai E, Lobrinus JA, Fallon BA, Tilton RC (2006) Lyme disease associated with Alzheimer's disease. Curr Microbiol 52, 330-332.

[5] Miklossy J, Kris A, Radenovic A, Miller L, Forro L, Martins R, Reiss K, Darbinian N, Darekara P, Mihaly L, Khalili K (2006) Beta amyloid deposition and Alzheimer's type changes induced by Borrelia spirochaetes. Neurobiol Aging 2, 228236.

[6] Pappolla MA, Omar R, Saran B, Andom A, Suarez M, Pavia C, Weinstein A, Shank D, Davis K, Borgdorfer W (1989) Concurrent neuroborreliosis and Alzheimer's disease: Analysis of evidence. Hum Pathol 20, 753-757.

[7] Miklossy J (2012) Chronic or late Lyme neuroborreliosis Analysis of evidence compared to chronic and late neurosyphilis. Open Neurol J 6, 146-157.

[8] Miklossy J (2011) Alzheimer's disease-a neurospirochetosis. Analysis of the evidence following Koch's and Hill's criteria. J Neuroinflammation $\mathbf{8}, 90$.

[9] Cummings JL (2011) Biomarkers in Alzheimer's disease drug development. Alzheimers Dement 7, e13-e44.

[10] O'Day DH (2013) The Alzheimer's Epidemic: Searching for Causes and a Cure. Emeritus Books, Oakville, 231.
[11] Canadian Lyme Disease Foundation (2013) Lyme disease, Alzheimer's disease, and inflammation-the relationship, http://canlyme.com/2013/01/28/lyme-disease-alzheimers-disease-and-inflamation-the-relationship/, Last updated January 28, 2013, Accessed on February 22, 2014.

[12] lymedisease.org (2011) HARDSCIENCEONLYME: Can Lyme disease cause Alzheimer's disease?, http://lymedisease org/news/hardscienceonlyme/802.html, Last updated August 25, 2011, Accessed on February 22, 2014.

[13] Tejada-Vera B (2013) Mortality from Alzheimer's disease in the United States: Data for 2000 and 2010. NCHS Data Brief No. 16, US Department of Health and Human Services 2013, http://www.cdc.gov/nchs/data/databriefs/db116.pdf, Accessed on February 22, 2014.

[14] Centers for Disease Control and Prevention.(2013) Reported cases of Lyme disease by state or locality, 2003-2012, http://www.cdc.gov/lyme/stats/chartstables/reportedcases_st atelocality.html, Last updated September 16, 2013, Accessed on February 22, 2014.

[15] Krut JJ, Zetterberg H, Blennow K, Cinque P, Hagberg L, Price RW, Studahl M, Gisslén M (2013) Cerebrospinal fluid Alzheimer's biomarker profiles in CNS infections. J Neurol 260, 620-626.

[16] Centers for Disease Control and Prevention (2013) Confirmed Lyme disease cases by age and sex-United States, 2001-2010, http://www.cdc.gov/lyme/stats/chartstables/incid encebyagesex.html, Last updated December 6, 2013, Accessed on February 22, 2014. 